\title{
A rare association of uterine leiomyoma with mesenteric vein thrombosis and bowel gangrene: case report
}

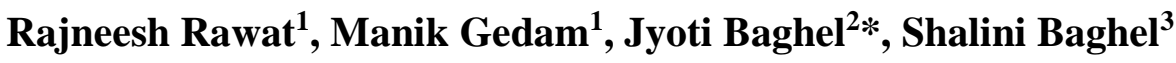

\author{
${ }^{1}$ Department of General Surgery, IGGMC, Nagpur, Maharashtra, India \\ ${ }^{2}$ Department of Obstetrics and Gynecology, AIIMS Nagpur, Maharashtra, India \\ ${ }^{3}$ Department of Obstetrics and Gynaecology, SN Medical College, Agra, Uttar Pradesh, India
}

Received: 05 February 2021

Revised: 09 March 2021

Accepted: 10 March 2021

\section{*Correspondence:}

Dr. Jyoti Baghel,

E-mail: bagheljyoti@aiimsnagpur.edu.in

Copyright: (c) the author(s), publisher and licensee Medip Academy. This is an open-access article distributed under the terms of the Creative Commons Attribution Non-Commercial License, which permits unrestricted non-commercial use, distribution, and reproduction in any medium, provided the original work is properly cited.

\begin{abstract}
Uterine fibroids are the most common benign pelvic tumors in women. There are many complications reported with fibroids. However, mesenteric vein thrombosis and small bowel gangrene caused by a uterine fibroid are rare. This manuscript reports a rare case of 40 year female with a large uterine fibroid associated with mesenteric vein thrombosis and bowel ischemia. She underwent exploratory laparotomy in which resection of gangrenous bowel including jejunum and ileum was done along with left sided jejunostomy and right sided ileostomy. Total abdominal hysterectomy with bilateral salpingooophorectomy was done followed by jejunoileal anastomosis 6 weeks later. Hence, in patients presenting with acute abdomen and uterine fibroids, bowel gangrene must be included in the differential diagnosis.
\end{abstract}

Keywords: Uterine leiomyoma, Acute abdomen, Portal vein thrombosis, Superior mesenteric vein thrombosis, Bowel gangrene, Case report

\section{INTRODUCTION}

Uterine fibroids (i.e. leiomyomata) are common benign smooth muscle tumors of the uterus. Most women will develop one or more uterine fibroids during their reproductive lifespan. ${ }^{1}$

Uterine leiomyomas are the most common pelvic tumors in women and occur in 20-30\% of women over 30 years of age. ${ }^{2}$ The fibroid may lead to various complications which include torsion, urinary retention, hemorrhage and thromboembolism. Thrombosis and subsequent embolic phenomenon due to large uterine fibroids is rarely reported complication. Here, we present a case report of large uterine leiomyoma causing portal vein, superior mesenteric vein thrombosis accompanied by bowel ischemia.

\section{CASE REPORT}

40 years female, homemaker, para 2, live 2, sterilized presented to emergency department with complaint of abnormal uterine bleeding since 5 days along with breathlessness and easy fatigability. She had complained of irregular menstrual cycles along with menorrhagia since last 6 months. This was her first ever visit to the hospital with the aforesaid complaint. She was admitted for evaluation of abnormal uterine bleeding in the ward. At night, she developed sudden onset of abdominal pain and hypotension

Her past medical history revealed no history of chronic diseases, no usage of oral contraceptive pills. She has no recent travel history and was not taking any medications. There was no family history of any thrombotic disorder. 
She was clinically very pale and overweight (BMI-28.6 $\mathrm{kg} / \mathrm{m}^{2}$ ). Her physical examination showed temperature of 98 degree fahrenheit, blood pressure $(\mathrm{BP})=90 / 50 \mathrm{mmHg}$, tachypnea (respiratory rate-30/min), tachycardia (heart rate $=130 \mathrm{bpm})$. Per abdominal examination revealed firm, irregular, mobile mass arising from the pelvis, corresponding in size to a pregnant uterus of 20-22 weeks. Diffuse tenderness over abdomen was present.

Laboratory analysis showed hemoglobin of $6.4 \mathrm{gm} / \mathrm{dl}$ with thrombocytosis (platelet count-5.2 lakh/ and normal serum electrolytes. D-dimer was found to be elevated (610 $\mathrm{ng} / \mathrm{ml}$ ). Transabdominal ultrasound revealed a large fibroid in the posterior uterine wall measuring $13 \times 11 \times 10$ $\mathrm{cm}$. There were features suggestive of portal vein thrombosis and superior mesenteric vein thrombosis. Also edematous bowel loops were seen with interbowel collection.

Contrast enhanced computed tomography (CECT) abdomen and pelvis revealed a large segment filling defect noted in the superior mesenteric vein suggestive of thrombosis. Similarly, filling defects were also noted in theportal vein and splenic vein suggestive of thrombosis .There was a large, well defined homogenous hypodense enhancing soft tissue density lesion of size $14.2 \times 11.4 \times 9.8$ $\mathrm{cm}$ along the posterior wall suggestive of uterine fibroid. Jejunal loops and proximal illeal loops appearedthickened and edematous with significantly reduced enhancement. These bowel loops appeared dilated with maximum dilatation of $4.2 \mathrm{~cm}$. Thus, the provisional working diagnosis was bowel gangrene secondary to mesenteric vein thrombus with uterine fibroid.
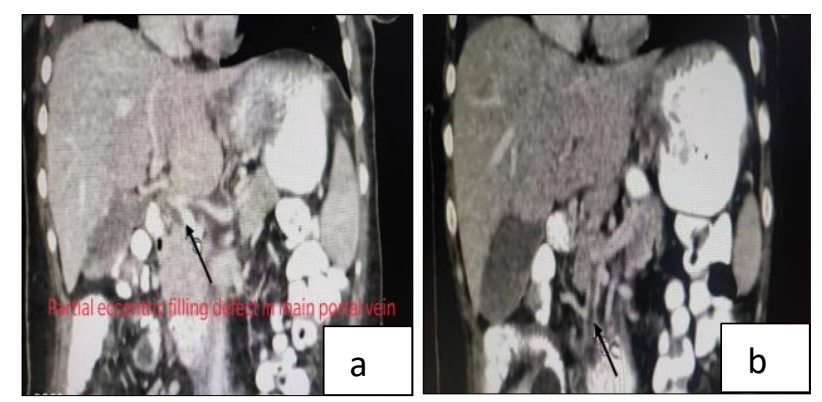

Figure 1: CT abdomen (a) partial eccentric filling defect in main portal vein and (b) complete non opacification of superior mesenteric vein (SMV thrombosis) [marked by an arrow].

Echocardiogram showed normal cardiac chamber dimensions, preserved systolic function, no pulmonary hypertension (pulmonary artery systolic pressure $=28$ $\mathrm{mmHg}$ ) and a normal left ventricular ejection fraction $(68 \%)$.

The patient was immediately taken for exploratory laparotomy with blood on flow. Written consent was taken for explorative surgery of the abdomen with the possibility of performing a hysterectomy. The patient was induced with spinal anesthesia and remained stable during the whole operation. Resection of gangrenous bowel including jejunum and ileum was done along with left sided jejunostomy and right sided ileostomy. Bowel resection included segment $30 \mathrm{~cm}$ away from duodenojejunal junction up to $20 \mathrm{~cm}$ of ileum proximally. Total abdominal hysterectomy with bilateral salpingooophorectomy was done.
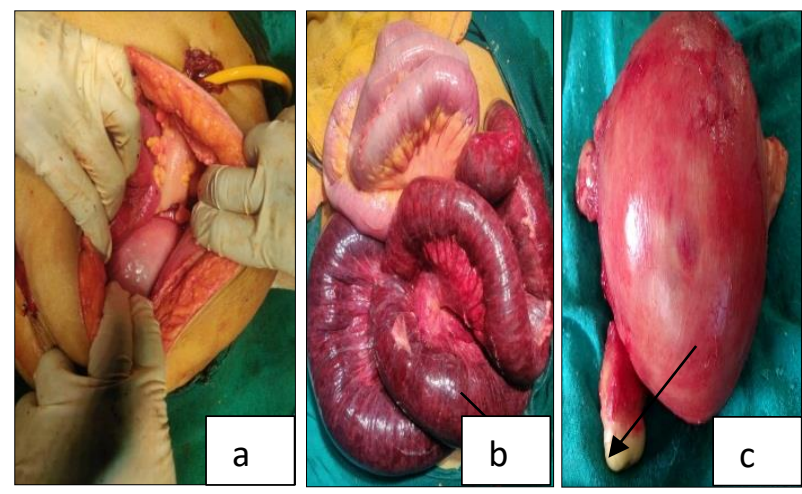

Figure 2: (a) Explorative laparotomy showing diffusely enlarged uterus in situ, (b) Picture showing distended and edematous segment of bowel loops and

(c) Specimen of total abdominal hysterectomy with bilateral salpingooophorectomy.

Due to prior severe anemia the patient was transfused with two units of blood intra operatively. A postoperative histopathological inspection showed the uterus to have intramural leiomyoma consisting of benign spindle cells, together with foci of necrosis and hematomas. A postoperative normalization of coagulation parameters and blood cell count occurred. She received enoxaparin injections in the post-operative period and was discharged on post-operative day 7. Again, after six weeks jejunoileal anastomosis was done. On regular follow-up examinations, the patient did not had any pathologies or complaints.

\section{DISCUSSION}

Uterine fibroids constitute as the most common benign pelvic tumor in women of the reproductive age group and areone of the major causes of abnormal uterine bleeding in the patients. ${ }^{2}$ Mostfibroids are asymptomatic; however, in 10 to $20 \%$ of the women they are symptomatic and require intervention. Clinical manifestations of uterine fibroids depend on their size, site, and their vicinity to adjacent structures and pelvic organs. Most common presentations include heavy menstrual bleeding, prolonged periods, pelvic pain and pressure symptoms.

Venous thrombosis due to large fibroids is a remote but documented complication. ${ }^{3-6}$ Mesenteric vein thrombosis and small bowel gangrene caused by a uterine fibroid infrequently reported in the literature. ${ }^{7}$ Various causes of thrombosis concomitant with fibroids have been proposed and are mainly associated with compression of local 
organs. In our present case, hepatic causes like cirrhosis or malignancies or extra hepatic causes of portal vein thrombosis were ruled out. Extrinsic obstructions and inherited hypercoagulable states were ruled out too. Sickle cell disease was also ruled out during work up.

Thrombus formation is governed by the principles of the Virchow's triad namelyendothelial injury, stasis and hypercoagulability. In large fibroid uterus, thrombus formation may be attributed to stasis of blood in the pelvic veins due to obstruction. It has been reported that iron deficiency anemia is associated with thrombosis of the cerebral veins and venous sinuses. Iron acts as a controller of thrombopoiesis and regulates the level of platelets. ${ }^{8}$ In iron deficiency the level of megakaryocytes increases, thereby increasing the platelet level, thus resulting in a hypercoagulable state. ${ }^{9}$ Since our patient had iron deficiency anemia, which might have induced a hypercoagulable state by altering pattern of blood flow. Further decreased hemoglobin levels may have resulted in poor oxygen supply to the tissues, leading to metabolic stress and endothelial damage. ${ }^{10}$

Recently it has been reported that there is increased thrombospondin-1 expression in uterine fibroid as compared to that seen in both normal endometrium and endometrial sarcoma. This thrombospondin is a glycoprotein involved in platelet adhesion reactions in addition to its role in inhibiting endothelial apoptosis. ${ }^{11}$ Also, it has been reported that uterine fibroid contains increased amounts of mRNA for basic fibroblast growth factor (fFGF) and exhibit abnormal expression of type 1 basic FGF receptor in the endometrium. ${ }^{12}$ This FGF receptor also plays a role of the heparin binding growth factor which might also interfere with normal coagulation.

There are a variety of treatments available - both medical and surgical. However, treatment modality depends on many factors such as size, number, site, symptoms, age of the patient, fertility status and comorbidities. In the present case bowel resection along with total abdominal hysterectomy with bilateral salpingooophorectomy was done in emergency.

\section{CONCLUSION}

In patients with an acute abdomen and uterine fibroids, gangrene of the intestine should be included in the differential diagnosis. Thus, every patient should be carefully evaluated so that the best multidirectional care may be provided timely and prevent serious complications.
Funding: No funding sources

Conflict of interest: None declared

Ethical approval: Not required

\section{REFERENCES}

1. Baird DD, Dunson DB, Hill MC, Cousins D, Schectman JM. High cumulative incidence of uterine leiomyoma in black and white women: ultrasound evidence. Am J Obstet Gynecol.2003;188(1):100-7.

2. Evans P, Brunsell S. Uterine fibroid tumors: diagnosis and treatment. Am Fam Physician. 2007;75:1503-8.

3. Sujindra E, Sabita SC, Bupathy A. Uterine myomatosis and portal vein thrombosis: a rare association. Int J Reprod Contracept Obstet Gynecol. 2016;5:3265-7.

4. Hawes J, Lohr J, Blum B, Bhati A, Bhaskaran J, Engel A. Large uterine fibroids causing mechanical obstruction of the inferior vena cava and subsequent thrombosis: a case report. Vasc Endovascular Surg. 2006;40:425-7.

5. Bonito M, Gulem L, Basili R, Brunetti G, Roselli D. Thrombosis associated with a large uterine myoma: case report. Clin Exp Obstet Gynecol. 2007;34:188-9.

6. Falcone M, Serra P. Massive pulmonary embolism in a woman with leiomyomatous uterus causing pelvic deep venous thrombosis. Annali Italiani di Medicina Interna. 2005;20:104-7.

7. Hamid H. Sheikh. A rare case of mesenteric vein thrombosis and small intestinal gangrene caused by a pedunculated fundal uterine fibroid is presented. The causative mechanism and management are discussed. Am J Obstet GynecoI. 1998;179:830-1.

8. Beguin Y. Erythropoietin and platelet production. Haematologica. 1999;84:541-7.

9. Karpatkin S, Garg SK, Freedman ML. Role of iron as a regulator of thrombopoiesis. Am $\mathrm{J}$ Med. 1974;57:521-5.

10. Balci K, Utku U, Asil T, Büyükkoyuncu N. Deep cerebral vein thrombosis associated with iron deficiency anemia in adults. J Clin Neurosci. 2007;14:181-4.

11. Bodner-Adler B, Nather A, Bodner $\mathrm{K}$, et al. Expression of thrombospondin 1 (TSP 1) in patients with uterine smooth muscle tumors: an immunohistochemical study. Gynecol Oncol. 2006;103:186-9.

12. Wolańska M, Bańkowski E. Fibroblast growth factors (FGF) in human myometrium and uterine leiomyomas in various stages of tumour growth. Biochimie. 2006;88:141-6.

Cite this article as: Rawat R, Gedam M, Baghel J, Baghel S. A rare association of uterine leiomyoma with mesenteric vein thrombosis and bowel gangrene: case report. Int J Reprod Contracept Obstet Gynecol 2021;10:1721-3. 